\title{
DIET AND EXERCISE KNOWLEDGE OF GESTATIONAL DIABETES OUTPATIENTS AT HANOI OBSTETRICS AND GYNECOLOGY HOSPITAL IN 2021
}

\author{
Nguyen Thi Thanh Tam', Le Thanh Tung', Mai Le Quyen², Dinh Thi Thu Huyen² \\ ${ }^{1}$ Hanoi Obstetrics and Gynecology Hospital; ${ }^{2}$ Nam Dinh University of Nursing
}

\begin{abstract}
Objective: To describe the knowledge about diet and exercise of gestational diabetes outpatients at Hanoi Obstetrics and Gynecology Hospital in 2021. Participants and methods: A cross-sectional descriptive study was conducted on 99 patients with gestational diabetes outpatient at Hanoi Obstetrics and Gynecology Hospital from February to April 2021. The study used the questionnaire of knowledge about the diet and exercise regime of people with diabetes with CVI specificity (0.97) and Cronbach alpha reliability (0.89). Results: Knowledge of diet and exercise regimen was good accounted for $9.1 \%$; quite good accounted for $39.4 \%$; average accounted for $30.3 \%$; poor accounted for $21.2 \%$. Conclusion: The patient's knowledge of diet and exercise regimen was still limited.

Keywords: Gestational diabetes, diet, exercise, knowledge.

\section{INTRODUCTION}

Gestational diabetes mellitus (GDM) is a glucose tolerance disorder of any degree, with onset or first detected during pregnancy [1]. Along with diabetes mellitus (DM), GDM is increasing rapidly worldwide, especially in developing countries as a result of urbanization, energy-rich food consumption, and lifestyle. The prevalence of GDM is increasing rapidly worldwide. This percentage ranges from $5.8 \%$ to $11.7 \%$ and varies among ethnic groups and regions [2]. GDM without timely intervention will cause many risks for mother and child such as preeclampsia, cesarean section due to large fetus, miscarriage, stillbirth, neonatal asphyxia, perinatal death.

Treatment of GDM includes a combination of methods: Nutrition, physical

activity, and medication, in which nutrition and exercise play a fundamental role. Many studies show that $70-85 \%$ of people with GDM can be treated with diet and exercise [3]. However, in Vietnam, studies on GDM mainly focus on the prevalence of GDM, but little research on nutritional knowledge and exercise regimes of GDM patients. Therefore, in order to assess the current status of knowledge for patients with GDM and as a premise for other hospitals to refer to, the researchers conducted a study on the topic "The diet and exercise knowledge of gestational diabetes outpatient at Hanoi Obstetrics and Gynecology Hospital in 2021". Objective: Describe the current status of knowledge about diet and exercise of gestational diabetes outpatients in Hanoi Obstetrics and Gynecology Hospital in 2021.
\end{abstract}

Cor. author: Nguyen Thi Thanh Tam

Address: Hanoi Obstetrics and Gynecology Hospital

Email: tam280682@gmail.com
Received: Feb 10, 2022

Accepted: Mar 03, 2022

Published: Mar 04, 2022 


\section{PARTICIPANTS AND METHODS}

\subsection{Participants}

Pregnant women were diagnosed with gestational diabetes treated as outpatients at the Nutrition Unit of Hanoi Obstetrics and Gynecology Hospital from February 2021 to April 2021.

\section{Inclusion criteria:}

Pregnant women were at 18 years old and more.

\section{Exclusion criteria:}

Pregnant women:

- Diabetes before pregnancy.

- Diagnosed with GDM from previous pregnancies.

- Placental-fetal abnormalities: placental abruption, intrauterine growth retardation.

-Malignancies, internal-surgical diseases, metabolic disorders, mental diseases, deaf-mute, and unable to communicate.

- Providing incomplete and inaccurate information, do not re-examine according to the appointment schedule.

\subsection{Research design}

Cross-sectional descriptive study.

\subsection{Sample size and sampling}

The whole sample method was used to collect 99 pregnant women.

\subsection{Measurements and data collection}

The measurement consisted of 2 parts:

Part 1: General information: Age, education level.
Part 2: Knowledge of diet and exercise regimen of pregnant women with GDM was developed based on the guidance of the document "National Guidelines on prevention and control of Gestational Diabetes" of the Ministry of Health in 2018 [4], "Guidelines for clinical nutrition treatment" of Bach Mai Hospital [5], "Clinical Nutrition of National Institute of Nutrition in 2019 [6], specificity CVI of 0.97; Cronbach's Alpha reliability of 0.89 . The questionnaire included 31 items with General knowledge (8 items), knowledge about carbohydrates regime (9 items), knowledge about fiber (4 items), knowledge about food processing (4 items), knowledge about exercise regime (6 items). Each correct answer of the pregnant women got 1 point, incorrect or unclear answers got 0 points. The total score of knowledge about diet and exercise regime was 31 points. Then, classify the patient's knowledge at 4 levels of good knowledge when the pregnant women answered correctly more than $80 \%$ of the total score; quite good knowledge when the pregnant women answered correctly $60 \%$ $79 \%$ of the total points; Average knowledge when the pregnant women answered correctly $50 \%-59 \%$ of the total score; Poor knowledge when the pregnant women answers correctly $<50 \%$ of the total score.

Data collection: The research team directly interviewed pregnant women.

\subsection{Data analysis}

The data were analyzed using SPSS 20.0 software. Using the calculation of the percentage (\%) of pregnant women according to the classification of knowledge levels, the percentage of pregnant women with correct knowledge according to each content. 


\section{RESULTS}

\subsection{Participant characteristics}

Table 1. Participant characteristics $(n=99)$

\begin{tabular}{|c|c|c|c|}
\hline \multicolumn{2}{|c|}{ Characteristics } & Number & Rate (\%) \\
\hline \multirow{4}{*}{ Age (years) } & $<25$ & 14 & 14,1 \\
\hline & $25-35$ & 71 & 71,1 \\
\hline & $>35$ & 14 & 14,1 \\
\hline & \multicolumn{2}{|c|}{ Mean \pm SD: $30,35 \pm 4,577$} & \\
\hline
\end{tabular}

Interpretation: Pregnant women were mainly at childbearing age 25-35 years old, accounting for $71.1 \%$.

Table 2. Characteristics of the educational level of the participants $(n=99)$

\begin{tabular}{|c|ccc|}
\hline \multicolumn{2}{|c}{ Characteristics } & Number & Rate (\%) \\
\hline \multirow{3}{*}{ Educational level } & Secondary school & 1 & 1,0 \\
\cline { 2 - 4 } & High school & 10 & 10,1 \\
\cline { 2 - 4 } & College & 15 & 15,2 \\
\cline { 2 - 4 } & University & 62 & 62,6 \\
\cline { 2 - 4 } & Post university & 11 & 11,1 \\
\hline
\end{tabular}

Interpretation: Pregnant women with a university degree accounted for $62.60 \% ; 1 \%$ of pregnant women had lower secondary education.

3.2. The knowledge about diet and exercise regimen of gestational diabetes women

Table 3. General knowledge of the diet of the participants $(n=99)$

\begin{tabular}{|lcc|}
\hline \multicolumn{1}{|c}{ Contents } & \multicolumn{2}{c|}{ Correct answers } \\
& Number & Rate (\%) \\
\hline Do you think a reasonable diet can stabilize blood glucose levels? & $\mathbf{5 0}$ & $\mathbf{5 0 , 5}$ \\
\hline Should you split your meals during the day? & 60 & 60,6 \\
\hline Eat 2 main meals and 3 snacks per day & 74 & 74,7 \\
\hline Eat whenever you feel hungry & 44 & $\mathbf{4 4 , 4}$ \\
\hline The amount of main meal food is equal to the amount of snack food & 62 & 62,6 \\
\hline Avoid eating a lot of animal organs & 58 & 58,6 \\
\hline Eat foods made from soybeans & 63 & 63,6 \\
\hline Drink at least 2000ml including water from food & 53 & 53,5 \\
\hline
\end{tabular}


Interpretation: The general knowledge about diet was quite low, with $44.4 \%$ of pregnant women eating whenever they feel hungry; $50.5 \%$ of participants had knowledge of a reasonable diet to stabilize glucose levels.

Table 4. Knowledge of carbohydrates in the diet of the participants $(n=99)$

\begin{tabular}{|lcc|}
\hline \multicolumn{1}{|c}{ Contents } & \multicolumn{2}{c|}{ Correct answers } \\
& Number & Rate (\%) \\
\hline Eat fewer carbohydrates (white rice, potatoes ..) & 53 & 53,5 \\
\hline Do not eat carbohydrates (white rice, potatoes ..) & 56 & 56,6 \\
\hline Eat white bread, vermicelli & 56 & 56,6 \\
\hline Eat black bread, brown rice, milled rice & 71 & 71,7 \\
\hline Drink lots of fruit juice & 60 & 60,6 \\
\hline Should eat a lot of sweet fruit (ripe jackfruit, ripe banana, ripe mango, ..) & 61 & 61,6 \\
\hline Should eat fruits with low sugar content: dragon fruit, orange, whip... & 64 & 64,6 \\
\hline Drink milk for pregnant women & 63 & 63,6 \\
\hline Drink milk with a low glycemic index: fresh milk-unsweetened yogurt... & 65 & 65,7 \\
\hline
\end{tabular}

Interpretation: The participants' knowledge about carbohydrates in the diet was still low, ranging from $53.5 \%$ to $71.7 \%$. In which, the participants had knowledge about reducing the number of carbohydrates by $53.5 \%$; eat black bread, brown rice, milled rice by $71.7 \%$.

Table 5. Knowledge of fiber in the diet of the participants $(n=99)$

\begin{tabular}{|llc|}
\hline \multicolumn{1}{|c}{ Contents } & \multicolumn{2}{c|}{ Correct answers } \\
& Number & Rate (\%) \\
\hline Eat a lot of vegetables in daily meals & 55 & 55,6 \\
\hline The amount of vegetables is equal to the food in the meal & 59 & 59,6 \\
\hline Eat vegetables after main meals (breakfast, lunch, dinner) & 59 & 59,6 \\
\hline Eat vegetables before main meals (breakfast, lunch, dinner) & $\mathbf{6 2}$ & $\mathbf{6 2 , 6}$ \\
\hline
\end{tabular}


Interpretation: $55.6 \%$ of participants had knowledge about eating a lot of vegetables in their daily meals and $62.6 \%$ of participants had knowledge about eating vegetables before main meals.

Table 6. Knowledge of food processing $(n=99)$

\begin{tabular}{|lcc|}
\hline \multicolumn{1}{|c}{ Contents } & \multicolumn{2}{c|}{ Correct answers } \\
& Number & Rate (\%) \\
\hline Processing boiled and steamed foods & 66 & 66,7 \\
\hline Processing fried and grilled foods & $\mathbf{6 8}$ & $\mathbf{6 8 , 7}$ \\
\hline Do not use animal fat in cooking & $\mathbf{5 7}$ & $\mathbf{5 7 , 6}$ \\
\hline Do not use vegetable oil in cooking & 63 & 63,6 \\
\hline
\end{tabular}

Interpretation: The participants' knowledge of food processing ranged from $57.6 \%$ to $68.5 \%$. In which, $57.6 \%$ of participants had correct knowledge about limiting the use of animal fat in food processing and $68.7 \%$ of participants had knowledge about frying and baking.

Table 7. Knowledge of the exercise regimen of the participants $(n=99)$

\begin{tabular}{|lcc|}
\hline \multicolumn{1}{|c}{ Contents } & \multicolumn{2}{c|}{ Correct answers } \\
& Number & Rate (\%) \\
\hline Exercise regime for diabetics effectively reduces blood sugar after eating & $\mathbf{5 0}$ & $\mathbf{5 0 , 5}$ \\
\hline $\begin{array}{l}\text { Choose exercises suitable for your health status, limit high-intensity } \\
\text { exercise movements. }\end{array}$ & $\mathbf{6 5}$ & $\mathbf{6 5 , 7}$ \\
\hline $\begin{array}{l}\text { The duration of each workout is about } 30 \text { minutes per day, most days } \\
\text { of the week. }\end{array}$ & 57 & 57,6 \\
\hline $\begin{array}{l}\text { Take precautions to prevent hypoglycemia while exercising. } \\
\text { Exercise before meals. }\end{array}$ & 63 & 63,6 \\
\hline Before, during, and after exercise, make sure to stay hydrated & 53 & 56,6 \\
\hline
\end{tabular}

Interpretation: Patients' knowledge about exercise was still quite low, ranging from $50.5 \%$ to $65.7 \%$. 
Table 8. Classification of knowledge about the diet and exercise regime of the participants $(n=99)$

\begin{tabular}{|lcccccccc|}
\hline \multicolumn{1}{c}{ Contents } & \multicolumn{2}{c}{ Good } & Quiet good & Average & \multicolumn{2}{c|}{ Poor } \\
& $\mathbf{n}$ & $\%$ & $\mathbf{n}$ & $\%$ & $\mathbf{n}$ & $\%$ & $\mathbf{n}$ & $\%$ \\
\hline General knowledge & $\mathbf{7}$ & $\mathbf{7 , 1}$ & 43 & 43,4 & 0 & 0 & $\mathbf{4 9}$ & $\mathbf{4 9 , 5}$ \\
\hline Knowledge about carbohydrates & 18 & 18,2 & 34 & 34,3 & 17 & 17,2 & 30 & 30,3 \\
\hline Knowledge about fiber & 19 & 19,2 & 28 & 28,3 & 0 & 0 & 52 & 52,5 \\
\hline Knowledge of food processing & 17 & 17,2 & 36 & 36,4 & 0 & 0 & 46 & 46,5 \\
\hline Knowledge of exercise & $\mathbf{2 3}$ & $\mathbf{2 3 , 2}$ & 24 & 24,2 & 0 & 0 & $\mathbf{5 2}$ & $\mathbf{5 2 , 5}$ \\
\hline Knowledge of diet and exercise & $\mathbf{9}$ & $\mathbf{9 , 1}$ & 39 & 39,4 & 30 & 30,3 & 21 & 21,2 \\
\hline
\end{tabular}

Interpretation: In general, the participants' knowledge about diet and exercise was still low, only $9.1 \%$ of the participants had good knowledge and $39.4 \%$ of the participants had good knowledge.

\section{DISCUSSION}

\section{Knowledge of diet and exercise regimen of participants}

The prenatal period in a pregnant woman's life is very important. For pregnant women with gestational diabetes, it is necessary to have knowledge about diet and exercise regimes to support the effective treatment of diabetes [7].

\subsection{Knowledge about the nutrition of the participants}

Diet is a measure to prevent and treat diabetes. Adherence to the diet helps patients maintain appropriate blood sugar levels, avoiding diabetes-induced complications [8].

The general knowledge of the participant's diet is quite low, with $44.4 \%$ of pregnant women eating whenever they feel hungry; $50.5 \%$ of participants had knowledge of a reasonable diet to stabilize glucose levels; $60 \%$ of participants had correct knowledge whether to divide meals during the day; $62.65 \%$ of participants had knowledge of food into 2 main meals and 3 snacks/day (Table 3). This result was higher than the study of Nguyen Trong Nhan, patients with knowledge of breakfast habits accounted for $57.1 \%$ and $33.7 \%$ of participants had correct knowledge of choosing the number of meals a day of diabetics [9]. This difference may be due to the different settings and research age in our study, the participants were pregnant women with diabetes, the average age was $30.35 \pm 4,577$, while the study Nguyen Trong Nhan, the participants were patients with diabetes in general with an average age of $58.88 \pm 11.47$ years old. 
People with diabetes should avoid large meals but divide them into several meals including 3 main meals, 1-3 snacks to help patients stabilize blood sugar, blood sugar does not rise too high after meals and does not fall too low when long from meals. In addition, they should not skip meals even if they do not want to eat because skipping meals can lower blood sugar or increase blood sugar after eating the next meal [8], [10].

Knowledge of carbohydrates in the patient's diet ranged from $53.5 \%$ to $71.7 \%$. In which, the participants had knowledge about reducing the number of carbohydrates by $53.5 \%$; eating black bread, brown rice, milled rice by $71.7 \% ; 61.6 \%$ of participants had correct knowledge about not eating a lot of sweet fruits (ripe jackfruit, ripe banana, ripe durian, ripe mango,..); $64.6 \%$ of participants with correct knowledge related to eating fruits with low sugar content: dragon fruit, oranges, whips (Table 4)... This result was higher than in the study Nguyen Thi Phuong Thuy that whole grains to replace rice were little known by pregnant women (accounting for 48.3\%) and are good foods for pregnant women at high risk of GDM. In particular, still, $21.7 \%$ of pregnant women think that dried fruit can be used for pregnant women at high risk of GDM [11].

As the American Diabetes Association (ADA) stated that as long as there are no allergies, all fruit is healthy, it is important to consider fruit preparation properly and advise people with diabetes should avoid drinking fruit juices or canned fruits with sugar because they are quickly absorbed leading to a rapid rise in blood sugar [12].

Knowledge about dietary fiber, 55.6\% of participants had knowledge about eating lots of vegetables in daily meals and $62.6 \%$ of participants had knowledge about eating vegetables before main meals (Table 5). This result was lower than that of Nguyen Le Huong's study, where $96 \%$ of pregnant women with GDM had correct knowledge about eating vegetables [14], and in Nguyen Thi Phuong Thuy's study, 73.3\% of participants had knowledge of different types of vegetables. High-fiber green vegetables in meals [11]. Thus, it can be seen that participants had low knowledge about dietary fiber. Why there was this difference, researchers should continue to research to find out more.

Green leafy vegetables contain many nutrients and antioxidants, which help protect eyes and heart health. Moreover, green vegetables contain fewer calories and starchy sugar, which helps limit blood sugar spikes. Some studies around the world showed that people with diabetes who absorb more vitamin $\mathrm{C}$ found in green vegetables will reduce inflammatory markers and make blood sugar rise more slowly. On the other hand, the fiber in fruits and vegetables is an important component in reducing sugar levels, slowing sugar absorption, and reducing postprandial hyperglycemia [8]. However, knowledge about the use of fiber in the diet of patients was still low. Therefore, this is the task that requires our research team to find out the knowledge gaps of the participants on this issue so that we can advise and overcome them to improve the participants' knowledge. According to the recommendations of the World Health Organization, providing increased fiber for people with diabetes is one of the very important principles in the treatment of diabetes with nutrition. Vegetables are a source of vitamins, minerals, fiber, and 
antioxidants for the body. Fiber makes starch stay in the stomach longer, reduces glucose absorption into the blood, has the effect of regulating blood glucose, so blood sugar does not rise suddenly. In addition, slow-digesting starches also create a feeling of fullness for a long time, contributing to soothing the blood sugar response.

Knowledge of food processing: $57.6 \%$ of participants had correct knowledge about using animal fat in food preparation and $68.7 \%$ of participants had correct knowledge about frying, grilling; $66.7 \%$ of the participants had knowledge about cooking steamed foods (Table 6). The results were different from the study of Nguyen Le Huong. $86.6 \%$ of participants had the knowledge to limit fatty foods; $87.1 \%$ of participants limited fried food [13]. In a study by Nguyen Trong Nhan, 42.9\% of participants had the correct knowledge to limit the use of fat [9].

This showed that the participants in our study did not pay enough attention to the diet, while the diet was an important part of the treatment strategy to control blood sugar as well as prevent complications of the disease. Therefore, nurses and health workers of the National Hospital of Obstetrics and Gynecology in particular and other facilities, in general, need to strengthen counseling on dietary knowledge for pregnant women with diabetes, especially diet counseling of fiber, in order to improve the participants' knowledge, thereby helping the patient to change the appropriate lifestyle, contribute to effective disease treatment and improve the health of the fetus.

\subsection{Knowledge of the training regimen of the DTNC}

Knowledge of exercise regimen: The participants' knowledge of exercise regime was still relatively low, in which, $50.5 \%$ of participants had knowledge about exercise regimen for diabetics that effectively reduces blood sugar after eating and $65.7 \%$ of participants had knowledge about choosing exercises suitable for their health status, limiting high-intensity exercise movements (Table 7). Exercise is necessary and good for everyone and especially good for people with diabetes. Regular exercise makes it easier for the body to consume sugar, increases energy consumption, reduces the risk of obesity, and reduces stress [8]. Therefore, the educational counseling on exercise regimens for pregnant women with diabetes by health workers is important in improving the knowledge of the participant's exercise regimen.

\subsection{Classification of knowledge about the diet and exercise regime}

The research results indicated that the knowledge about diet and exercise regimes of the study participants was relatively low. Of 99 patients, $9.1 \%$ of participants had good knowledge about nutrition and exercise; $39.4 \%$ of participants had quite good knowledge; $30.3 \%$ of participants had average knowledge, and $21.2 \%$ of participants had poor knowledge. In which, participants had good knowledge of sugar accounting for $18.2 \%$; fiber accounting for $19.2 \%$; food processing accounting for $17.2 \%$; exercise regimen accounting for $9.1 \%$.

A lack of knowledge about diet and exercise can lead to a number of dangerous complications: Increased blood glucose levels or greatly reduced blood glucose levels, hypoglycemia situations, retinal hemorrhages conjunctivitis, ischemic heart disease, or sudden death [14]. The low knowledge about the nutrition and exercise 
regime of pregnant women with GDM will seriously affect the treatment process of the disease of pregnant women and seriously affect the health of the fetus. Therefore, counseling to improve knowledge for participants with GDM is necessary.

\section{CONCLUSION}

Research on 99 people with GDM obtained results that the patient's knowledge about diet and exercise regimen was still low:

General knowledge was $7.1 \%$ at good; $43.4 \%$ at quite good ; $0 \%$ at average; $49.5 \%$ at poor.

Knowledge of carbohydrates was $18.2 \%$ at good; $34.3 \%$ at quite good; $17.2 \%$ at average; $30.3 \%$ at poor.

Fiber knowledge was $19.2 \%$ at good; $28.3 \%$ at quite good; $17.2 \%$ at average; $52.5 \%$ at poor.

Processing knowledge was $17.2 \%$ at good; $36.4 \%$ at quite good; $0 \%$ at average; $46.5 \%$ at poor.

Knowledge of exercise regime was $23.2 \%$ at good, $24.2 \%$ at quite good; $0 \%$ at average; $52.5 \%$ at poor.

Knowledge of diet and exercise regimen was $9.1 \%$ at good; $39.4 \%$ at quite good; $30.3 \%$ at average; $21.2 \%$ at poor.

From this result, health workers need educational counseling on diet and exercise regimens for pregnant women with GDM. In particular, there is a need for regular interventions on the importance of a healthy diet, eating fiber and reducing carbohydrates, and ensuring rehydration before, during, and after exercise.

\section{REFERENCES}

1. American Diabetes Association (ADA) (2017). Standards of medical care in diabetes. Classification and Diagnosis of Diabetes. Diabetes Care. 40 (1), pp. 40. https://doi.org/10.2337/cd16-0067

2. Y. Zhu, C. Zhang (2016). Prevalence of Gestational Diabetes and Risk of Progression to Type 2 Diabetes: a Global Perspective. Curr Diab Rep. 16(1), pp. 7.https://link.springer.com/article/10.1007/ s11892-015-0699-X

3. Huynh Nguyen Khanh Trang and Truong Thi Nguyen Hao (2017). Effect of dietary restriction on pregnant women with gestational diabetes at Thu Duc district hospital. Ho Chi Minh Practical medicine. 21(1), p. 80-85.

4. Ministry of Health (2018). National guidelines on gestational diabetes, issued together with Decision No. 6173/QD-BYT dated October 12, 2018, of the Minister of Health

5. Luong Ngoc Khue and Nguyen Quoc Anh (2015). Guidelines for clinical nutritional therapy, Medical Publishing House.

6. Le Danh Tuyen, Nguyen Thi Lam and Pham Thi Thu Huong (2019). Clinical Nutrition, Medical Publishing House, Hanoi.

7. Nabila E. Saboula, et al (2018). Effect of Nursing Intervention on Knowledge, Attitude and Self -Care Activities among Gestational Diabetic Women. International Journal of Novel Research in Healthcare and Nursing. Vol. 5, Issue 2, pp: (135-146), Available at: www.noveltyjournals.com

8. Ta Van Binh (2001). People with diabetes need to know, Medical Publishing House. 
9. Nguyen Trong Nhan (2019). Changing knowledge and dietary practices of type 2 diabetes outpatient after health education at Bac Giang Province Endocrinology Hospital in 2019. Master's of nursing thesis , Nam Dinh University of Nursing.

10. Nguyen Huy Cuong (2008). Outline of diabetes. Diabetes - modern perspectives. Medical Publishing House.

11. Nguyen Thi Phuong Thuy (2021). Survey of knowledge on prevention of gestational diabetes among pregnant women in Nam Dinh Obstetrics and Gynecology Hospital in 2021. Master's of nursing thesis, Nam Dinh University of Nursing.
12. American Association of Clinical Endocrinologists and American College of Endocrinology (2015). Clinical practice guidelines for developing a diabetes mellitus comprehensive care plan, Edition. https://doi.org/10.4158/EP15672.GL

13. Nguyen Le Huong (2012). Knowledge, practice, and prevalence of GDM among pregnant women at National Hospital of Obstetrics and Gynecology in 2012 and related factors.

14. Ruderman N., et al. (2002). Diabetes and exercise: the risk-benefit profile revisited and the exercise prescription. Handbook of exercise in diabetes (2nded.). Alexandria,, American Diabetes Association. 